OPEN ACCESS

Edited by:

Luis Eduardo Antunes Vieira, National Institute of Space Research (INPE), Brazil

Reviewed by: Juan Carlos Martínez Oliveros, University of California, Berkeley, United States Grzegorz Michalek, Jagiellonian University, Poland

*Correspondence: Angelos Vourlidas angelos.vourlidas@jhuapl.edu

Specialty section

This article was submitted to Stellar and Solar Physics,

a section of the journal Frontiers in Astronomy and Space

Sciences

Received: 20 April 2020

Accepted: 16 June 2020

Published: 14 August 2020

Citation:

Vourlidas A, Carley EP and Vilmer N (2020) Radio Observations of Coronal Mass Ejections: Space Weather Aspects.

Front. Astron. Space Sci. 7:43 doi: 10.3389/fspas.2020.00043

\section{Radio Observations of Coronal Mass Ejections: Space Weather Aspects}

\author{
Angelos Vourlidas ${ }^{1 *}$, Eoin P. Carley ${ }^{2}$ and Nicole Vilmer ${ }^{3,4}$ \\ ${ }^{1}$ Applied Physics Laboratory, Johns Hopkins University, Laurel, MD, United States, ${ }^{2}$ School of Cosmic Physics, Dublin \\ Institute for Advanced Studies, Dublin, Ireland, ${ }^{3}$ LESIA, Observatoire de Paris, Université PSL, CNRS, Sorbonne Université, \\ Université de Paris, Meudon, France, ${ }^{4}$ Station de Radioastronomie de Nançay, Observatoire de Paris, PSL Research \\ University, CNRS, Univ. Orléans, Nançay, France
}

We review the current state-of-affairs in radio observations of Coronal Mass Ejections (CMEs) from a Space Weather perspective. In particular, we examine the role of radio observations in predicting or presaging an eruption, in capturing the formation stages of the CME, and in following the CME evolution in the corona and heliosphere. We then look to the future and identify capabilities and research areas where radio observations - particularly, spectropolarimetric imaging - offer unique advantages for Space Weather research on CMEs. We close with a discussion of open issues and possible strategies for enhancing the relevance and importance of radio astronomy for Space Weather science.

Keywords: sun, radio astronomy, coronal mass ejections, space weather, spectropolarimetry

\section{INTRODUCTION}

The modulation of the near-Earth space environment by solar activity, over short time scales (days or less), is known as Space Weather (SpWx). The modulation, particularly when it is impulsive and sustained, can have severe effects on space-borne civil and military systems (e.g., satellite operations, communication disruptions) and even on the lower atmosphere and ground (e.g., aviation and electric grids). As our society increasingly depends on those systems, concern on SpWx impacts rises, spurring research and strategy planning worldwide (Schrijver et al., 2015; Opgenoorth et al., 2019). The latest demonstration of the societal importance of SpWx is the publication of the Space Weather Strategy and Action Plan (SWAP) by the Office of Science and Technology Policy (OSTP).

The strongest SpWx effects are caused by the impact of Coronal Mass Ejections (CMEs) and their shocks onto the magnetosphere. The CME momentum, size, and magnetic field strength and configuration, are the most relevant physical parameters for energy input to geospace. Irradiance variations from flares cause perturbations in deeper atmospheric layers while solar energetic particles (SEPs) are a major concern for any human exploration to the Moon and beyond. Mitigation of SpWx impacts relies, at the moment on forecasting primarily CME impacts (and their associated phenomena; shocks and SEPs). Flare short-wavelength, soft X-rays (SXR) to ultraviolet (UV), emissions and intense radio bursts (IRBs) that disrupt radio communications including GPS systems, are currently impossible to forecast because they arrive at Earth nearly instantaneously (i.e., within $8 \mathrm{~min}$ of their occurrence on the Sun). In addition, the causes of IRBs are currently unknown, they may or may not occur with other eruptive activity, and their terrestrial impacts can be severe. They are discussed in more detail in Gary (2020).

Their extreme nature aside, IRBs show that radio observations have an important role in both $\mathrm{SpWx}$ research and operations. Radio emission arises from a broad range of physical phenomena 
with SpWx implications (e.g., flares, SEPs, CMEs and shocks). The observations, and theoretical background, are reviewed in other chapters of the collection. Here, we revisit the phenomena associated with CMEs from a SpWx viewpoint and with emphasis on the CME propagation from the middle corona to $1 \mathrm{AU}$. We consider $3 \mathrm{R}_{s}$ as the inner boundary of the middle corona, because it is the approximate height of the cusps of white light streamers and where the magnetic field becomes largely open to the heliosphere. The CME-related radio emissions for lower heights are discussed in Carley et al. (2020b). As the first paper within the Space Weather section, we take a more broad view of radio CMEs and the role of radio observations in the SpWx enterprise. The remaining chapters focus on radio effects that are not strictly related to CMEs, such as SEPs (Klein et al., 2020), IRBs (Gary, 2020) and interplanetary scintillation (IPS) techniques (Jackson et al., 2020).

The paper is organized in three sections. First, we summarize the status of the field around the three SpWx-relevant phases of eruptions (prediction, formation and propagation) with an emphasis on the propagation phase. Then, we give an outlook of the radio observing capabilities (with emphasis on imaging spectroscopy) and the SpWx-areas where radio can play an important or supporting roles. We close with a discussion of considerations and strategies for maximizing the SpWx potential of radio observations in the coming decade.

\section{RADIO AND CMES: CURRENT STATUS}

Several of the chapters in this book review or discuss the observations and emission processes related to CMEs and associated phenomena, mostly from a research perspective. In this section, we bring them together but organized around a SpWx framework. Specifically, we break down radio CME observations into three SpWx areas (in parentheses): (i) observations before the eruption (event prediction), (ii) observations during the eruption (event geoeffectiveness assessment), and (iii) observations after the eruption (event forecasting). To minimize repetition, we direct the reader to the appropriate references for the details, except where necessary for clarity.

\subsection{Before the Eruption}

Robust prediction of eruptive activity is considered by many the "holy grail" of SpWx research and unsurprisingly is a very active field, focused mostly on flare prediction based on metrics derived from observations of the photospheric magnetic fields (e.g., Leka et al., 2019). Predicting CME eruptions, however, is a very complex issue. While we understand that CMEs are driven by the explosive release of magnetic energy through generally identified physical mechanisms that result in the release of a magnetic flux rope (MFR) (Chen, 2011), the details of how magnetic systems destabilize and erupt escape us. A rather major problem is that CMEs are coronal phenomena but we do not have direct measurements of the coronal magnetic field. We are obligated to resort to proxies, such as photospheric magnetic field measurements or changes in the EUV or SXR emission from coronal structures. Consequently. there is yet no agreement on whether the pre-eruptive morphology is that of sheared magnetic arcades or of an MFR (see Georgoulis et al., 2019, and references therein). Thus, our physical understanding is not yet mature enough to lead to reliable predictions schemes for solar eruptions. Here is where radio observations may help bridge our knowledge gap, either as indications of an impending eruption (precursors) or as gauges of energy accumulation and imbalance in active regions (predictors). We discuss examples of both.

\subsubsection{Precursors: Type-I Noise Storms and Other Emissions}

Radio emission occurs throughout the solar atmosphere, from the chromosphere to interplanetary space (IP), via a variety of processes (Fleishman et al., 2020; Nindos et al., 2020) all arising from electrons, either in equilibrium or not (Figure 1). Non-thermal processes are of particular interest in our discussion because they arise from accelerated electrons and therefore indicate locations of energy release in the corona. Even mildly accelerated electrons of a few $\mathrm{keV}$ can emit detectable radio emission, making such observations a sensitive indicator of weakly energetic processes. Hence, their detection in subsequently-erupting regions could be precursor activity. This premise and the indications from models that the pre-eruptive magnetic field configuration is evolving as the system is driven toward loss of equilibrium, have spurred several efforts to identify radio CME precursors (for a discussion on CME precursors in other regimes see section 3.9 in Webb and Howard, 2012).

The promising candidates are Type-I noise storms, type-III bursts and certain radio continuum signatures. Type-I storms are thought to indicate energetic processes within closed magnetic loops. These may be high-rising loops, as the storms occur below $300 \mathrm{MHz}$ generally. The emission usually arises from loop systems above magnetically-strong active regions and is well-associated with flux emergence. Its relation to flaring and erupting activity is more tenuous. There have been a few studies since 2005 (see Vourlidas 2004 for a review of earlier studies). Willson (2005b) discusses radio brightness changes in a TypeI storm ahead of a flare but in another case, they find no connection between the two (Willson, 2005a). Kathiravan et al. (2007) undertook a large-scale study of noise storms imaged with the Nançay Radioheliograph. They investigate only noise storms with start times after the onset of a CME. They identified 196 events (out of a total of 340) in 1997-2004, with an average delay of $13 \mathrm{~h}$ since the appearance of a CME in the LASCO coronagraph field of view. Ramesh et al. (2012a) analyzed a single noise storm imaged with the Gauribidanur Radioheliograph (GRH) in the 50-109 $\mathrm{MHz}$ range (corresponding to heights below 1.5 Rs for quiescent coronal density models) during a solar eclipse (Figure $\mathbf{2 b}$ ). The storm was concurrent with the CME but it was located almost $180^{\circ}$ from the CME position angle in the LASCO coronagraph. Neither of these studies provided any conclusive evidence for the relation between CMEs and noise storms and they certainly did not indicate that noise storms can be used as precursors. So we have no reason (yet) to change our original conclusion in (Vourlidas, 2004): "It seems that the two phenomena are somehow interrelated but the details of the relationship (physical, temporal and/or spatial) are still unclear". 


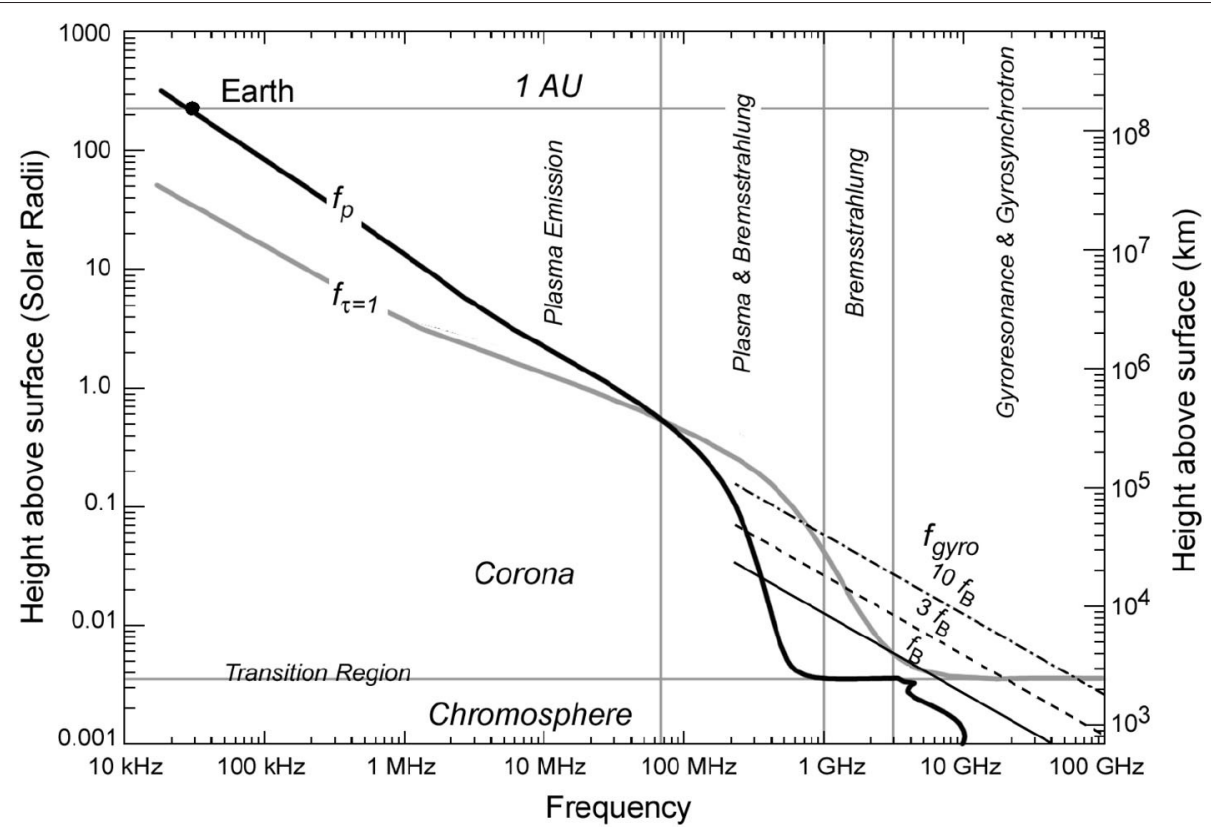

FIGURE 1 | Dominant radio emission mechanism vs. heliocentric distance based on model plasma parameters of temperature, density, and magnetic field strength. $f_{p}$ are the plasma frequency, $f_{B}$ is the gyrofrequency, and $f_{\tau=1}$ is the frequency where free-free emission becomes optically think. For details on these quantities and physical mechanisms (see Alissandrakis et al., 2020; Nindos et al., 2020). The plot is available here (Courtesy D. Gary).
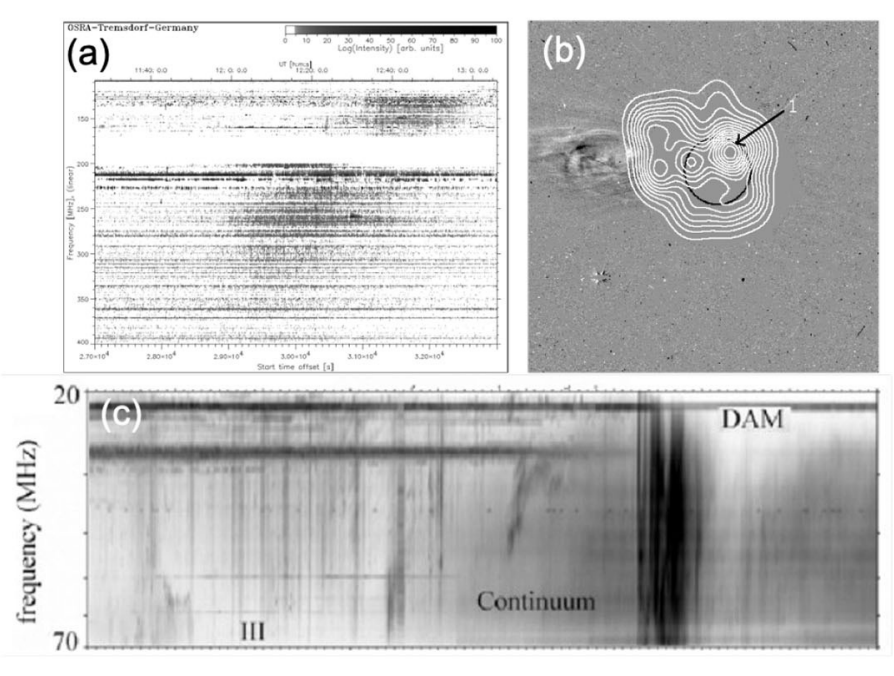

(d)
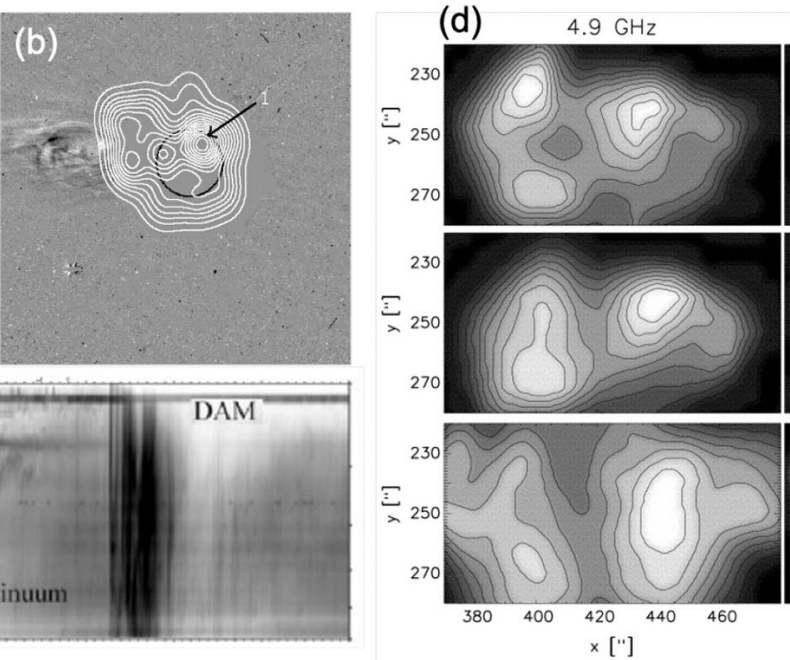

$8.4 \mathrm{GHz}$
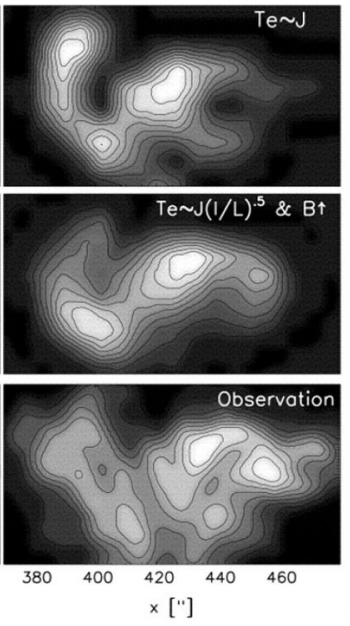

FIGURE 2 | (a) Astrophysical Institute Potsdam spectra showing the faint type IV continuum on May 16 (from Aurass et al., 1999). (b) Composite of the GRH $109 \mathrm{MHz}$ radioheliogram (white contours) at 05:00 UT and the SOHO-LASCO C2 image at 05:19 UT on 2010 January 15 showing the CME and Type-I noise storm (from Ramesh et al., 2012b). (c) DAM radio spectrum of an outburst following type-IIl emissions and a continuum increase (from Pick et al., 2005). (d) Comparison of model (top two rows) and observed (bottom row) radio intensities at 4.9 and $8.4 \mathrm{GHz}$ (left and right panels, resp.). The key assumption for the dependence of electron temperature to currents is shown at the upper right-hand corner of the right panels. The comparison demonstrates the power of multi-frequency observations in constraining coronal magnetic properties (from Lee et al., 1998). All figures reproduced by permission of the AAS.

Type-III emission is another promising precursor candidate as it arises from outward propagating electron beams and may thus indicate recently opened field lines. Such magnetic field changes are postulated by CME initiation models, such as breakout (Antiochos et al., 1999) and are generally expected as the CME forms and begins to emerge from within the closed fields of active regions. Based on our review of type-IIIs in the low corona in Carley et al. (2020b), we conclude that although such emissions are detected frequently in eruptive events, they tend to occur in close temporal proximity $(\sim \operatorname{mins})$ to the flare impulsive phase 
and are of little value as a precursor (Pohjolainen et al., 2005; Aurass et al., 2011, 2013). The question, however, remains on whether earlier type-III signatures exist that may have escaped detection because either they are weak and may be masked by other activity or because no one has searched specifically for them.

Finally, there is another type of radio signature, the rising continuum, first proposed as a precursor by Aurass et al. (1999) (Figures 2a,c). Detected also $30 \mathrm{~min}$ before the large eruption on Oct 28, 2003 (Figure 2 and Pick et al., 2005), this feature has attracted little attention in the recent years. Detections have been recorded only within the decimetric range, a few $10^{5} \mathrm{~km}$ above the surface and so may indeed be indications of the rising flux system before eruption. More observations are sorely needed to demonstrate the value of this radio feature.

Fortunately, the deployment of new radio instrumentation looks promising. Mugundhan et al. (2018) reports results on several types of noise storms from a high resolution spectropolarimeter in the $15-85 \mathrm{MHz}$ range at Gauribidanur. McCauley et al. (2019) present similar polarization investigations from the MWA at $80-240 \mathrm{MHz}$. It is hoped, that as the activity picks up the increased sensitivity and frequency complementarity of these two instruments will shed some light on the relation between noise storms and CME onset (or aftermath, as the case might be).

\subsubsection{Predictors: Energy Accumulation in ARs}

CMEs are coronal phenomena. They are powered by the release of free magnetic energy and helicity in the corona (Georgoulis et al., 2019). The energy is accumulated and stored in stressed magnetic field and should therefore reveal itself by measuring currents in those fields. Alas, the coronal magnetic field is notoriously difficult to measure (e.g., Casini et al., 2017) and there is currently no monitoring capability as it exists for photospheric magnetic fields. Almost all radio emission mechanisms have a magnetic field diagnostic potential since they tend to arise from electron gyration around magnetic field lines. The potential has been demonstrated numerous times (see White, 2005; Casini et al., 2017; Alissandrakis et al., 2020, and references therein). Spectropolarimetric imaging of gyroresonance emission is one of the most straightforward options for deriving 3D distributions of magnetic field from the upper chromosphere to the corona (e.g., Vourlidas et al., 1997). These studies occasionally uncover $\mathrm{kG}$ fields at coronal temperatures within $\sim 3,000 \mathrm{~km}$ above the photosphere (e.g., Vourlidas and Bastian, 1996; Vourlidas et al., 2006) suggesting the presence of strong currents at the cores of active regions (Lee et al., 1997, 1998) (Figure 2d).

There is, however, a catch. Robust detection and separation of gyroresonance harmonic emission is limited to strong fields (down to $\sim 100 \mathrm{G}$ as explained in Casini et al., 2017). In other words, gyroresonance emission as a magnetograph proxy is suitable to active region observations only. This is not necessarily overly restrictive from a SpWx perspective, since the most geoeffective CMEs are expected to originate (and they do) from strongly magnetic active regions.

\subsection{During the Eruption}

There is a great host of radio emissions during the formation phase of the CME, which we review in Carley et al. (2020b). They are associated with flares, lifting of prominences, and opening of field lines, and can be readily understood within the context of the standard flare-CME model (see Figure 1 in Carley et al., 2020b). In fact, much of that connection has been apparent for a long time and there have been no surprises despite the great improvements in the sensitivity and cadence of radio observations since the 1990's (Figure 3). There is a slight exception, however; the detection of "radio" CMEs by the Nancay Radioheliograph (NRH; Kerdraon and Delouis, 1997). The fortuitous coincidence of NRH's imaging capability deployment with LASCO's start of science operations in 1996, led to the discovery of spatially resolved (but faint) radio emission within the white light transient, dubbed "radio" CMEs because of their similar appearance to LASCO CMEs (Bastian et al., 2001). There have been only two such "radio" CME detections (Bastian et al., 2001; Maia et al., 2007) since 1998 despite the rather continual coverage from imaging spectrometers, i.e., NRH, LOFAR, and MWA. We note here that the term "radio" CMEs tends to be broadly applied to events without a clear CME morphology (e.g., Carley et al., 2017; Mondal et al., 2019). The latter are actually more akin to moving Type-IV (mIV) bursts (section 2.2 and Carley et al., 2020b) and may also have a different emission mechanism (plasma rather than gyrosynchrotron). However, "radio" CMEs may just be spatially resolved mIVs but the reason behind the dearth of "radio" CME detections remains unclear. More details can be found in Carley et al. (2020b).

The SpWx relevance of "radio" CMEs and mIVs lies in their emission mechanism. If it is synchrotron, as commonly assumed in the analyses (e.g., Tun and Vourlidas, 2013; Sasikumar Raja et al., 2014; Carley et al., 2017; Mondal et al., 2019), then their detection provides a means to estimate the (total) magnetic field entrained in the CME, while the CME is still in the low corona. Such information could help improve prediction schemes of the geoeffectiveness of the transient when augmented, say, with 3D estimates of the CME volume and its evolution from white light coronagraphs. At the moment, all methods for predicting the CME magnetic field rely on observations and extrapolations of the photospheric field (e.g., Gopalswamy et al., 2017; Savani et al., 2017), which are just proxies. Of particular value is the southward component of the CME entrained magnetic field, $B_{Z}$, at $1 \mathrm{AU}$ as it relates directly to the strength of the CMEmagnetosphere interaction (see Vourlidas et al., 2019 for a review of the $B_{Z}$ problem). The radio observations offer a unique way to estimate the total magnetic field (and $B_{Z}$ if the CME magnetic configuration is assumed or modeled) close to the Sun and issue some sort of forecast with a day (or possibly more) horizon rather than waiting for the transient to cross the Lagrangian L1 point thus restricting the forecast horizon to an hour or less. One should consider the intervening evolution of the CME as interactions with upstream events (some of which have radio signatures, e.g., Gopalswamy et al., 2001) or the ambient solar wind can lead to field erosion or compression depending on the situation (Kilpua et al., 2019). 


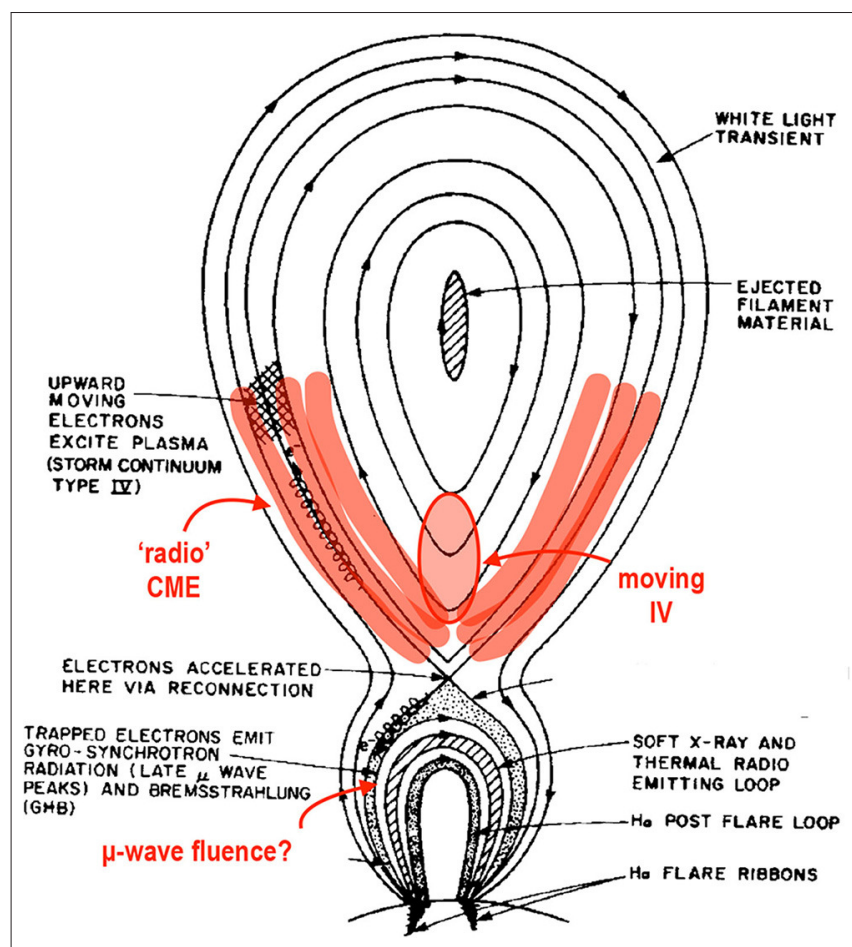

FIGURE 3 | A cartoon representing the flare-CME model from Cliver et al (1986) showing the origin of various radio emissions. We mark areas impacted by the newer observations. Reproduced by permission of the AAS.

We conclude that spatially-resolved radio observations of CMEs hold a rich, yet unfulfilled, potential for SpWx research and operations. Their sensitivity of radio observations to magnetic field across a wide swath of features, from quiescent active regions to flares to $\mathrm{CME}$ internal structures, makes them the ideal method for probing the geo-effectiveness of CMEs right "at birth." But that potential is not restricted to the low corona, as we shall see next.

\subsection{After the Eruption: CME Propagation}

One of the outstanding issues in SpWx operations is the accurate forecasting of the time-of-arrival (ToA) of a CME at Earth. There are many methods to forecast ToA (most recently reviewed in Vourlidas et al. 2019) varying widely in accuracy. The averaged (over most published research) mean absolute error in ToA currently stands at $9.8 \mathrm{~h}$, which is too large to be useful for many SpWx users. Vourlidas et al. (2019) discusses several reasons for this discrepancy and they largely revolve around our incomplete grasp of the IP kinematic evolution of the CMEs. The speed of CMEs is especially difficult to assess for the most SpWxrelevant events-Earth-directed CMEs-due to severe projection effects in the low corona (EUV) and middle corona (visible) observations. To circumvent these problems, several authors have searched for emission signatures concurrent to CMEs, such as EUV dimmings (Mason et al., 2016), soft X-ray light curves and more recently high frequency $(\mathrm{GHz})$ fluence measurements, to use as proxies by relating their evolution to the $\mathrm{CME}$ speed via empirical relationships. Focusing on the radio proxies, Matamoros et al. (2017) have recently demonstrated a correlation between microwave fluence at $9 \mathrm{GHz}$ and $\mathrm{CME}$ arrival time at the Earth, using limb CME observations to construct the empirical (linear in this case) relationship. The performance of the method is average and has only been demonstrated with a very small event sample (11 events) so it is unclear if it holds SpWx potential. Further work to increase the event sample and possibly examine other frequencies is necessary.

Once the CME reaches 3-4 $\mathrm{R}_{s}$, it enters into the radial (open) field corona and effectively starts its outward propagation toward the inner heliosphere. This height range marks a transition in our abilities to observe CMEs in the radio from the ground, as the relevant frequencies dip below $20 \mathrm{MHz}$ and encounter the ionospheric cutoff. Routine lower frequency observations can be made only from space. Space radio spectrometers can use direction finding techniques (Fainberg et al., 1972; Krupar et al., 2012) to track type II sources in $3 \mathrm{D}$ via triangulation, when radio spectra from two spacecraft are available (e.g., Magdalenić et al., 2014; Krupar et al., 2016; Mäkelä et al., 2018). As the accuracy of the technique depends on the source signal-to-noise ratio, the instrument cross-calibration and the relatively wide directivity of type-II radio emission, the ensuing localizations are rather broad (of the order of 10-20 $\mathrm{R}_{s}$ ) and are generally restricted to nearSun tracking ( $\mathrm{MHz}$ frequencies). The complexity of the analysis has restricted the application of radio triangulation to only a handful of events despite the availability of measurements from three spacecraft (STEREO, Wind) since 2007. In any case, the rather large uncertainties in the source location and size limits the Space Weather utility of radio triangulation compared to direct imaging in white light. No radio imaging capability currently exists but there are two space interferometry pathfinders under development. The Cubesat Radio Interferometry Experiment (CURIE, Sundkvist et al., 2016), in development since 2018, aims to demonstrate single-baseline interferometry $(0.1-40 \mathrm{MHz})$ of solar bursts (centroid location and envelope) using 2 cubesats in Low Earth Orbit. The mission is not yet manifested. Recently, NASA selected Sun Radio Interferometer Space Experiment (SUNRISE, Lazio and Kasper, 2018), an imaging interferometry space mission comprising six CubeSats in super-GEO orbit with a launch in late 2023. SUNRISE's aim is to image and localize type-II bursts below $25 \mathrm{MHz}$. While these frequencies can track CMEs only up to $20 \mathrm{R}_{s}$, and hence do not provide much additional information beyond what is currently available from coronagraphs, the mission will demonstrate whether solar interferometric imaging is possible in space. We will return to this point in section 3.3.

Before the advent of heliospheric imaging from the SolarTerrestrial Relations Observatory (STEREO) (Harrison et al., 2018), tracking of CMEs in the outer corona and heliosphere had been possible only from space-borne spectrometers albeit indirectly. The tracking was based on the detection of typeII emission arising from shocks driven by CMEs, not CME themselves (see Figure 4 and Cane and Erickson, 2005 for more examples). Occasionally, the type-II spectral signatures extend to the local plasma frequency around the detecting spacecraft, hence enabling track to $1 \mathrm{AU}$. The IP type-II sources, although 

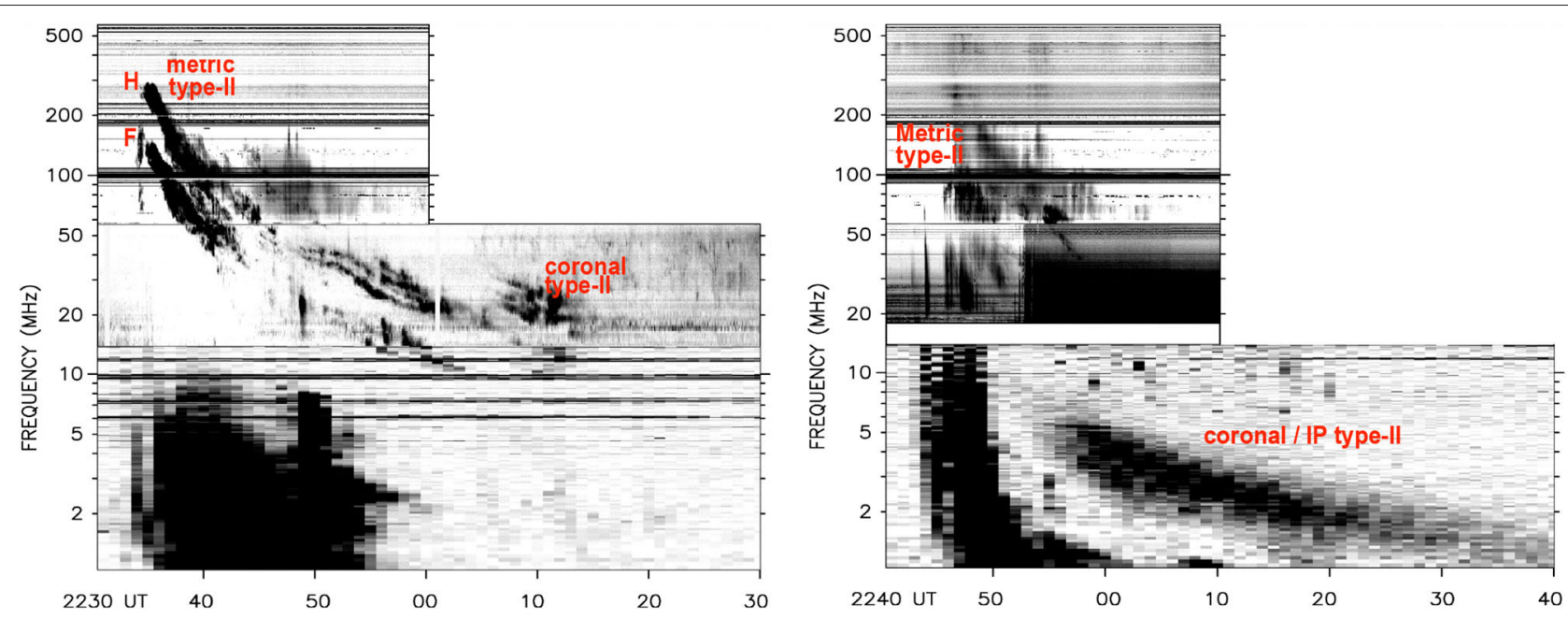

FIGURE 4 | Examples of type-II bursts in the low corona ("metric") outer corona ("coronal") and IP. Left: Composite spectrum on 2003 November 1. The WAVES data are below $14 \mathrm{MHz}$, the BIRS data run from 14 to $57 \mathrm{MHz}$, and the Culgoora data run from 57 to $570 \mathrm{MHz}$. Fundamental ("F") and harmonic ("H") bands of the type II are easily seen, along with band-splitting. A short, type II-like feature ("coronal") is seen in the BIRS data between 23:06 and 23:14 UT. There is no indication of an IP counterpart. Right: The beginning of an IP type-II on 2003 June 17 22:40 UT. It is apparently unconnected to the metric type-II seen in the higher frequencies. Culgoora data are used between 18 and $57 \mathrm{MHz}$. Adapted from Cane and Erickson (2005). Reproduced by permission of the AAS.

similar in many respects to their lower corona counterparts, are much longer-lived (hours to days compared to a few minutes) and are unambiguously driven by CMEs (Pick et al., 2006 and references therein). There is long-standing ambiguity regarding the connection between the coronal and IP shocks (Leblanc et al., 2001; Cane and Erickson, 2005; Bougeret and Pick, 2007 and Figure 4, right panel) although the differences between metric and IP type-IIs may simply be due to the shocks being produced at different phases of the CME evolution; namely, the metric bursts originate during the over-expansion phase of the CME formation (Patsourakos et al., 2010) while IP type-IIs are due to shocks driven by the largely self-similarly expanding CME (Cane and Erickson, 2005).

The main SpWx benefit of IP type-II observations is the quasi-continuous tracking of the CME shock in the outer corona/inner heliosphere. The shock can be variously driven or free-propagating depending on the CME kinematical behavior and the upstream conditions in the ambient medium. Thus, type-IIs should be treated as a (possibly rough) proxy for the actual CME. Unfortunately, such long-lived IP type-II's are uncommon since they require CMEs capable of shock-driving over large parts of their propagation to $1 \mathrm{AU}$. Gopalswamy et al. (2019) undertook recently a thorough statistical investigation of IP type-IIs detected by the Wind spacecraft since 1995. They find only about 500 events, which correspond to a very small fraction $(3.1 \%)$ of all CMEs. Only half of those bursts reached below $500 \mathrm{KHz}$. On the positive side, these bursts are associated with faster CMEs $(1,160 \mathrm{~km} / \mathrm{s}$ average speed), which tend to have higher geoeffective potential. While the radio spectroscopic measurements are unaffected by particle storms that may blind a coronagraph using a traditional CCD detector (this will no longer be the case after the deployment of NOAA's operational coronagraphs at L1 and GEO in 2024) and are straightforward to interpret and to derive a speed, they have some serious impediments for SpWx use. IP type-IIs are not a robust indicator of a fast CME (low association with white light CMEs, not all fast CMEs drive shocks or have radio emissions, Gopalswamy et al., 2010), they track the shock rather than the transient and the tracking is incomplete (only 250 bursts in the last 25 years have reached near 1 AU locations). However, the IP radio observations are highly complementary to heliospheric imaging and provide key information on IP shocks, which constitute SpWx hazards of their own.

Radio observations, offer the only means to probe the CME internal magnetic field during heliospheric propagation via linear polarization measurements (Jackson et al. 2020, and references therein). The linearly polarized emission from an extragalactic (or artificial source, say, a satellite beacon) rotates when crossing magnetized plasma. The effect is called Faraday Rotation (FR) and the degree of rotation, known as Rotation Measure (RM), depends on the total density and magnetic field along the path (e.g., Kooi et al., 2017 and references therein). The left and middle panels of Figure 5 show recent FR observations by the VLA of not one but two CMEs crossing over the radio source 0843 (Kooi et al., 2017). The modeling was able to disentangle the RM contribution along the line-of-sight of each transient demonstrating that the technique can be successful in estimating the magnetic field of CMEs in the outer corona. As CMEs carry, generally, higher magnetic fields than the ambient heliosphere and intergalactic space, they will be the dominant contributors to the measured FR (Figure 5, right and Oberoi and Lonsdale, 2012). If the density of the transient is known, say, from white light measurements, then the total magnetic field within the CME (along a given path) can be estimated. Modeling of the CME magnetic structure can be employed to derive the magnetic field configuration (Jensen et al., 2010; Le Chat et al., 2014), 

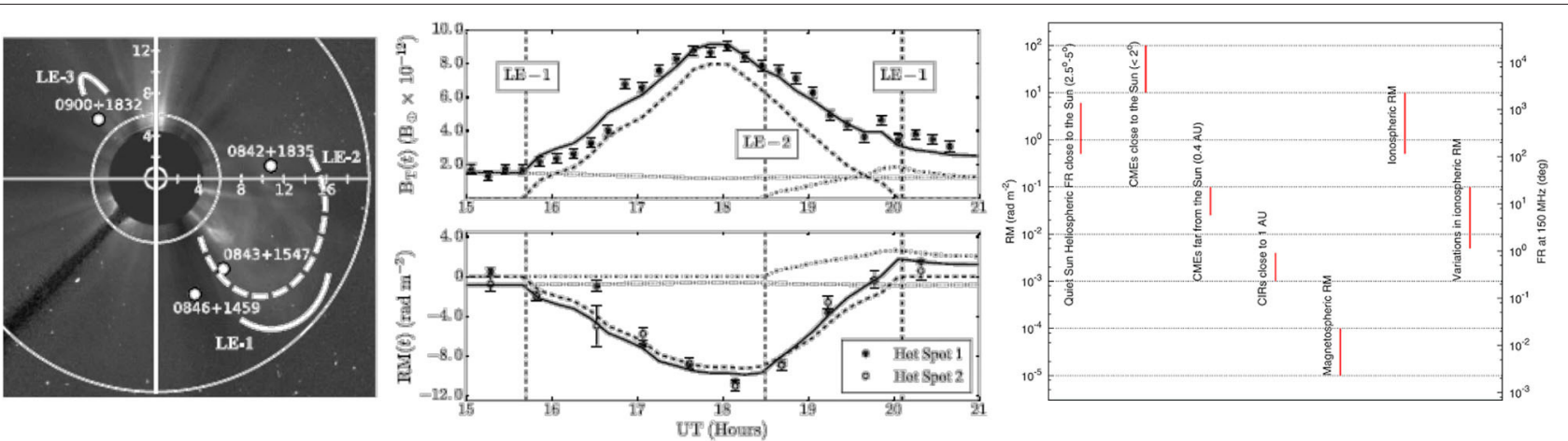

FIGURE 5 | Left: LASCO observations at the time (19:06 UT) of the 0843 radio source occultation by two CMEs (CME-1 and CME-2) on Aug. 2, 2012. Middle: Thomson-scattering brightness (top) and coronal RM (bottom) for 0843. The Thomson brightness is given for one line-of-sight to the target source center (see Kooi et al., 2017 for details on Hot Spot 1 and 2). The dotted curve represents the background coronal model, the dashed curve represents the flux-rope model for CME-1, the dash-dotted curve represents the flux-rope model for CME-2, and the solid curve represents the sum of the contributions from all models together. The LE-1 vertical lines mark the times of the radio source occultation by CME-1 (15:42 UT and 20:06 UT, respectively). LE-2 marks the start of CME-2 occultation (18:30 UT) (Adapted from Kooi et al., 2017 with permission by Springer Nature). Right: Overview of the RM effects from various plasmas. The corresponding FR (in deg) at 150 $\mathrm{MHz}$ is also indicated. $\mathrm{A} \pm$ range of a factor of 2 has been assumed where needed. Adapted from Oberoi and Lonsdale (2012) with permission by the AGU.

which is actually the critical parameter for SpWx. There are other indirect methods for estimating the CME magnetic field (Kilpua et al., 2019, and references therein) but FR remains the most straightforward technique.

It does, however, have its shortcomings. For one, it requires the presence of a suitable source (i.e., a well-calibrated celestial or artificial source) at the right place to intersect the CME while the radio instrument is operating. This is a set of very strong requirements and it is unsurprising that only a handful of measurements have been recorded since the 1970's (see Kooi et al., 2017 for a historical review). Furthermore, the measurements are biased toward limb CMEs as they provide a wider projection on the sky plane and thus are more likely to intercept a celestial source. The technique applies to Earthdirected CMEs, of course, but those lines of sight will cross the shock sheath as well the magnetic flux rope behind it, increasing the ambiguity in the decoupling of line-of-sight effects and thus relying more on modeling assumptions. In any case, the SpWx potential of this technique has not been demonstrated yet since it would require both continuous coverage from Earth, and a large selection of calibrated sources. No FR measurements have been demonstrated in space. The optimum SpWx location for such a receiver would likely be one of the Lagrange point to monitor CMEs on the sky plane as they propagate toward Earth. A receiver-transmitter system on concurrently-operating satellites at L4/L5 could be the basis for a SpWx-operational system to provide $B_{z}$ measurements of Earth-bound transients.

\section{OUTLOOK}

So far, we reviewed CME aspects where radio observations can contribute to problems in SpWx research and operations. Hopefully, our discussion (and the accompanying papers in this ebook) makes clear that solar radio astronomy, particularly spectropolarimetric imaging, has great potential to impact SpWx research by closing several gaps in our knowledge, from the magnetic field distribution in pre-eruptive active regions to the magnetic field and energetic particle content of the transients.

In this last section, we take a strategic look in the near future of radio CME research, always from a SpWx perspective. We first take stock of the available instrumentation, then summarize and assess the relative importance of the various radio emission types for CME SpWx research and close with a brief discussion of some issues to consider for moving the field forward.

\subsection{Future Radio Instrumentation Relevant to CME SpWx Studies}

Developments in radio instrumentation in the present day and those planned for the near future promise to provide new insight into CME physics. The Expanded Owens Valley Solar Array (EOVSA) is now providing unprecedented imaging-spectroscopy observations of the early stages of energy release and CME formation, low in the corona $\left(<3 \mathrm{R}_{\odot}\right)$. The observations tend to focus on the sources of non-thermal electrons associated with the eruption, such as in the current sheet and the associated termination shocks. They are therefore indirectly connected to SpWx concerns on CMEs but they usher a new understanding of the eruptive process as a whole that may provide SpWx benefits down the line.

New instruments such as MingantU SpEctral Radioheliograph (MUSER; Mei et al., 2018) will have an ultrawide bandwidth from $400 \mathrm{MHz}$ to $15 \mathrm{GHz}$, bridging the gap between the microwave and lower frequency instruments and should provide a more comprehensive view of radio sources associated with both flares and CMEs. Although not specifically geared toward space weather observations, future radio facilities such as the Square Kilometer Array (SKA) will deploy ground-breaking new instrumental capabilities on general flare/CME physics in a wideband observing range from $300 \mathrm{MHz}$ to $14 \mathrm{GHz}$; see Nindos 
et al. (2019) for an overview of solar radio physics from an SKA perspective.

Lower frequency observations from instruments such as the Low Frequency Array (LOFAR; van Haarlem et al., 2013) and the Murchison Widefield Array (MWA; Li et al., 2018) can provide CME diagnostics generally $\leq 3 R_{\odot}$, via imaging observations of type II, III and IV radio bursts during the acceleration phase of the CME (e.g., Zucca et al., 2018; Mondal et al., 2019; Morosan et al., 2019). Such observations enable an independent measure of ejecta speed while the CME is still in the low corona, where its early stage propagation may be obscured behind the occulting disks of coronagraphs. As we mentioned, if the CME can be directly imaged in the radio domain (which is still a rarity) it may be used to perform CME magnetography. We expand in Carley et al. (2020b) on the necessity of high dynamic range interferometric imaging for observing radio CMEs, and efforts in this regard have recently been demonstrated with MWA leading to spatially resolved diagnostics of magnetic field of the ejecta (Mondal et al., 2019).

Low frequency phased-array interferometers such as LOFAR and MWA attain their most powerful SpWx utility when they are used as beamformers on multiple background sources to perform IPS observations. This requires observation of well-calibrated astrophysical radio sources. Beamformers, such as LOFAR and MWA, can simultaneously observe multiple astrophysical radio sources (potentially hundreds) in many different directions and hence can provide IPS diagnostics over large portions of the inner heliosphere. Hence, IPS can be used to derive density and velocity measurements in the solar wind, including any CME that is passing through the heliosphere, and can therefore be used as a means of estimating the arrival time of Earth directed CMEs (Bisi et al., 2010). This may also be used as boundary conditions in the driving of MHD models of the heliosphere such as ENLIL (Jackson et al., 2015). Apart from MWA and LOFAR, phased arrays are now used to perform routine observations of the heliosphere for space weather purposes e.g., the Mexican Array Radio Telescope (MEXART; Mejia-Ambriz et al., 2010) operating at $140 \mathrm{MHz}$, KSWC-IPS in Korea (327 MHz), the Solar Terrestrial Environment Laboratory ISEE IPS array (Asai et al., 1995) in Japan $(327 \mathrm{MHz}$ ), the Big Scanning Array of the Lebedev Physical Institute (Dagkesamanskii, 2009) in Russia (110 MHz), and the Ooty Radio Telescope (ORT; Sukumar et al., 1988) operating at $327 \mathrm{MHz}$ in India. These arrays are grouped together into a consortium of IPS monitors known as Worldwide Interplanetary Scintillation Stations, aiming to provide $24 \mathrm{~h}$ space weather monitoring coverage of the heliosphere and any CME passing through it (see Jackson et al., 2020 for details).

Related to IPS are the Faraday rotation (FR) measurements of radio emission passing through the heliosphere (Jensen et al., 2010), as discussed in section 2.3. Studies are currently under way to assess the requirements of modern instrumentation in observing FR, particularly at low radio frequencies where the ionosphere can contribute to the rotation measure significantly (Figure 5). A new project known as LOFAR for Space Weather (LOFAR4SW; Carley et al., 2020a) is a design study to upgrade the entire LOFAR system such that it provides routine observation of the Sun, heliosphere, and ionosphere from a space weather science and operations perspective. The upgraded system aims to perform daily imaging spectroscopy of the Sun, including imaging of the radio activity during the early phases of solar eruptions, as well as IPS and FR observations of the solar wind and CMEs propagating throughout the heliosphere.

Finally, space-based radio instrumentation is also entering a new era. The FIELDS instrument (PSP-FIELDS; Bale et al., 2016) on-board the recently launched Parker Solar Probe and Radio and Plasma Waves (RPW; Maksimovic et al., 2007) instrument on Solar Orbiter will provide a new perspective on the observation of radio bursts in the inner-heliosphere from $10 \mathrm{kHz}$ to $20 \mathrm{MHz}$. However both are primarily research instruments, rather than platforms offering the continuous lowlatency observations required by space weather operations. As mentioned above, SUNRISE will also expand upon low radio frequency observations of the heliosphere, offering for the first time the ability to observe radio bursts interferometrically and provide $2 \mathrm{D}$ positional information with the constraints discussed in section 2.3 .

\subsection{An Assessment of Radio CME Observations for SpWx Research}

We have presented a relatively large number of radio emission types and studies in this and our companion paper (Carley et al., 2020b). Although they provide important information for physical properties and processes in quiescent and eruptive phenomena, they do not all have the same impact when viewed from a SpWx viewpoint. Some parameters, e.g., CME occurrence or speed, can be provided by other instrumentation, often in a much more continuous and robust way compared to the limited daily operations of most radio observatories. However, other information, such as the CME internal magnetic field can only be derived synoptically through radio observations.

In Table 1, we organize the various radio emissions into a SpWx-relevant list. We mark as "essential" the types of observations (column 1) that are unique to radio and provide highly sought-after parameters for $\mathrm{SpWx}$ research and operations (columns 2-3). We also assess the top-level instrumental capabilities required to maximize the SpWx benefit (column 4). We consider the required (required: "Y" or not: "N") spectral (Sp), imaging (Img), and polarimetric (Pol) capabilities but we do not discuss any specific frequency range or spatial/spectral resolution requirements. These details, as well as a broader SpWx perspective are summarized in a White Paper by Bastian et al. (2019). The "?" denotes areas where the SpWx value of a particular observation or capability is not obvious at the moment. Further research with a possibly stronger SpWx focus may be useful for those areas.

\subsection{Considerations for Moving Forward}

With a new solar cycle on the horizon, improvements in groundbased instrumentation and an ever-increasing societal attention to the Space Weather problem, it is time to consider the future of Radio SpWx research. To increase the value of ground- and space-based radio observations to $\mathrm{SpWx}$ research and operations, we first need to consider some strategic questions. For example, where do we focus instrument development efforts? which 
TABLE 1 | Overview of radio emission types with relevance to SpWx CME research.

\begin{tabular}{|c|c|c|c|c|c|c|}
\hline \multirow[t]{2}{*}{$\begin{array}{l}\text { Emission } \\
\text { type }\end{array}$} & \multirow[t]{2}{*}{$\begin{array}{l}\text { SpWx } \\
\text { concern }\end{array}$} & \multirow[t]{2}{*}{$\begin{array}{l}\text { SpWx } \\
\text { parameters }\end{array}$} & \multicolumn{3}{|c|}{$\begin{array}{l}\text { Instrumental } \\
\text { capabilities }\end{array}$} & \multirow[t]{2}{*}{ Remarks } \\
\hline & & & Sp & Img & Pol & \\
\hline AR mapping & Prediction & 3D mag. field, coronal currents & Y & Y & Y & Essential capability for assessing AR eruptive potential. SpWx potential \\
\hline "Radio" CMEs & $\mathrm{B}_{Z}$ & CME mag. field strength & Y & Y & Y & Essential capability for mag. field estimates. SpWx potential \\
\hline Moving Type-IV & $\mathrm{B}_{Z}$ & CME mag. field strength & Y & Y & Y & Essential capability for mag. field estimates. SpWx potential \\
\hline Faraday Rotation & $\mathrm{B}_{Z}$ & $\begin{array}{l}\text { ICME mag. field strength \& } \\
\text { configuration }\end{array}$ & $\mathrm{N}$ & N & Y & Essential capability for remote $B_{Z}$ estimate. SpWx potential. \\
\hline Type-॥ & Shock tracking, SEP & Shock speed & Y & $?$ & $\mathrm{~N}$ & IP type-Ils are useful but no robust association to CMEs \\
\hline Type-I & Precursor? & CME onset & Y & $?$ & Y & Unclear connection to $\mathrm{CME}$ \\
\hline Microwave fluence & CME Speed & CME TOA & Y & $\mathrm{N}$ & $N$ & Promising approach. Needs more research \\
\hline Flare Continuum & SEP? & CME kinematics? & Y & Y & Y & Unclear connection to $\mathrm{CME}$ \\
\hline
\end{tabular}

science activities are likely to return the most impactful SpWxrelevant results? The answers should come from a communitywide discussion. To get the conversation going we present a few issues (some may be controversial) we derived during the compilation of our two reviews (Carley et al. 2020b and the present manuscript). We note again that the following issues pertain to SpWx aspects of CMEs.

- Radio as a CME Magnetograph: We believe that the most important SpWx value of radio observations is their unique ability to estimate magnetic fields both in active regions and inside CMEs. High-frequency arrays $(\mathrm{GHz})$ are best suited for coronal magnetic field mapping, especially for eruptionprone ARs (e.g., $\delta$-spots). Lower frequency arrays $(<200$ $\mathrm{MHz}$ ) are ideal for IVM and "radio" CME imaging. High signal-to-noise ratio imaging is paramount (Bastian et al., 2019). The SpWx value can only be realized when those measurements are available synoptically and as close to $24 \times$ 7 , as possible. Continuous solar coverage is a fundamental need for operational SpWx. Even for research purposes, nearly continuous coverage will greatly increase the numbers of all radio emission types and further their understanding. In other words, we need the deployment of solar-dedicated interferometric arrays with spectropolarimetric capabilities across the globe.

- How necessary are IP radio observations for CME-specific SpWx issues? The case is not very strong. As Gopalswamy et al. (2019) show, only a very small fraction of CMEs (3.1 are associated with IP type-II bursts. Those tend to be fast CMEs but it is also clear that a number of fast CMEs either do not drive shocks in the heliosphere or their radio emission is too faint or shocks do not always produce radio emission (Gopalswamy et al., 2008). The bottom line is that type-II bursts cannot be a reliable CME proxy on their own. Of course, this statement refers to CMEspecific SpWx issues, such at the CME time-of-arrival, speed and magnetic content (Vourlidas et al., 2019). IP shocks can be a SpWx driver but they are beyond the scope of this review (see Kilpua et al., 2017 and references therein for a broader review on this subject).

A question arises on whether imaging of IP (or at least of the outer corona) type-IIs has something more to offer on predicting either CMEs or shock properties at $1 \mathrm{AU}$ (Gopalswamy et al., 2010). The problem is that radio sources tend to be very large due to scattering. Therefore, any imaging reduces to centroid localization that may or may not be accurate since the type-II emission depends on the ambient microphysics (that are not well-understood, although PSP and Solar Orbiter may shed some light on this). It is unclear (at best) that imaging at low frequencies will provide any SpWxrelevant information that cannot be gleaned by coronagraphs or heliospheric imagers.

- Are there any radio CME precursors?: This is an important question for $\mathrm{SpWx}$ operations. No reliable precursor to CMEs has been identified so far in any wavelength. Most CME initiation theories require magnetic field line opening above (and/or the sides) of the CME flux rope as it forms and begins to ascend. It is, therefore, expected that these field line openings, resulting from reconnection, would release energetic electrons and hence some radio beaming signature is expected. Recent PSP observations detected weak type-III signatures in association with small surges on the solar disk (Leske et al., 2020). The PSP was located at about 30-40 $\mathrm{R}_{s}$ at the time and in alignment with STEREO-A, which did not detect any radio emission. It is conceivable, then, that CME precursors may exist in the radio but have escaped detection due to the high detection threshold of past instruments. For instance, it would be interesting, to investigate whether such weak radio signatures could arise in radio quiet CMEs (Gopalswamy et al., 2008) or from "stealth" CMEs (Robbrecht et al., 2009) that generally lack low corona signatures in other spectral regimes. On the other hand, there are rare instances where major flares (but no CMEs!) have taken place without any radio emission. Since, however, all of these cases involve non-eruptive flares, they are not relevant for $\mathrm{SpWx}$ (except for the flare radiation effects). A search for such precursors may prove more fruitful with MWA and LOFAR in the upcoming maximum.

- What to do next?: It seems to us that there is considerable potential for SpWx-relevant studies now by taking advantages of existing ground-based instrumentation and missions. For example, the frequent crossings of PSP and of Solar Orbiter 
through the corona provide an excellent opportunity for coordinated Faraday rotation, mIV and "radio" CMEs studies with LOFAR, MWA and EOVSA. Solar radio observatories were the biggest ground-based component in the campaign to support the fourth PSP perihelion in January 2020. Upwards of 20 solar radio observatories participated and results are already being compiled even before the PSP data are processed. We expect that radio observations will play an increasing role in PSP and Solar Orbiter research.

Careful design and planning of SpWx-specific observing programs will maximize the $\mathrm{SpWx}$ benefit of radio observations. The LOFAR4SW project mentioned above is an example (Carley et al., 2020a). It comprises a set of observing programs for diverse targets, such as CME Faraday rotation studies, multi-spectral imaging or IPS observations. The coordinated campaigns with PSP perihelia we just mentioned offer another opportunity for developing such programs, which can then be used outside of the PSP or Solar Orbiter perihelia for a more SpWx-minded efforts as the activity picks up.

Radio analyses should also adopt a more SpWx viewpoint. For example, large sample analyses of type-I storms and mIVs or searches for "radio" CMEs and more focus on the derivation of magnetic fields in CMEs may increase the visibility of radio observations within the SpWx community. Further research to identify radio proxies for CME SpWx parameters, such as speed, may prove valuable. Regular availability of wellcalibrated radio images at various wavelengths, as it is done by the solar space missions, will help greatly in increasing

\section{REFERENCES}

Alissandrakis, K., et al. (2020). Radio measurements of solar magnetic field. Antiochos, S. K., DeVore, C. R., and Klimchuk, J. A. (1999). A model for solar coronal mass ejections. Astrophys. J. 510, 485-493. doi: 10.1086/306563

Asai, K., Ishida, Y., Kojma, M., Maruyama, K., Misawa, H., and Yoshimi, N. (1995). Multi-station system for solar wind observations using the interplanetary scintillation method. J. Geomagnet. Geoelectricity 47, 1107-1112. doi: 10.5636/jgg. 47.1107

Aurass, H., Holman, G., Braune, S., Mann, G., and Zlobec, P. (2013). Radio evidence for breakout reconnection in solar eruptive events. Astron. Astrophys. 555:A40. doi: 10.1051/0004-6361/201321111

Aurass, H., Mann, G., Zlobec, P., and Karlický, M. (2011). Radio evidence of break-out reconnection? Astrophys. J. 730:57. doi: 10.1088/0004-637X/730/1/57

Aurass, H., Vourlidas, A., Andrews, M. D., Thompson, B. J., Howard, R. H., and Mann, G. (1999). Nonthermal radio signatures of coronal disturbances with and without coronal mass ejections. Astrophys. J. 511, 451-465. doi: 10.1086/306653

Bale, S. D., Goetz, K., Harvey, P. R., Turin, P., Bonnell, J. W., Dudok de Wit, T., et al. (2016). The FIELDS instrument suite for solar probe plus. Measuring the coronal plasma and magnetic field, plasma waves and turbulence, and radio signatures of solar transients. Space Sci. Rev. 204, 49-82. doi: 10.1007/s11214-016-0244-5

Bastian, T., Bain, H., Chen, B., Gary, D. E., Fleishman, G. D., Glesener, L., et al. (2019). Diagnostics of space weather drivers enabled by radio observations. arXiv [Preprint]. arXiv:1904.05817.

Bastian, T. S., Pick, M., Kerdraon, A., Maia, D., and Vourlidas, A. (2001). The coronal mass ejection of 1998 April 20: direct imaging at radio wavelengths. Astrophys. J. Lett. 558, L65-L69. doi: 10.1086/323421 the community uptake of radio observations from the new instruments. Such capabilities should be in place before the next solar maximum in about 2024 for maximum effect for SpWx research.

In closing, we emphasize once more the great potential of ground-based spectropolarimetric radio imaging for addressing important open questions in SpWx research. Radio imaging of moving Type-IVs and "radio" CMEs with Faraday Rotation measurements in the outer corona, offers a straightforward means to estimate the CME's internal magnetic field and possibly configuration, with the aid of modeling. The construction of a solar-dedicated instrument, such as FASR and further efforts like LOFAR4SW, will energize the international radio community and provide an ideal testbed to refine and expand the $S p W x$ value of radio observations.

\section{AUTHOR CONTRIBUTIONS}

EC and NV contributed text and figures. All authors contributed to the initial concept, design of the paper, read and critically revised the paper, approved the final version, and agreed to be accountable for all aspects of the work.

\section{FUNDING}

AV was supported by the NASA HSR (NNX16AG86G) and LWS (80NSSC19K0069) programs. EC is a Shrödinger Research Fellow funded by the Dublin Institute for Advanced Studies.

Bisi, M. M., Breen, A. R., Jackson, B. V., Fallows, R. A., Walsh, A. P., Mikić, Z., et al. (2010). From the Sun to the Earth: the 13 May 2005 coronal mass ejection. Solar Phys. 265, 49-127. doi: 10.1007/s11207-010-9602-8

Bougeret, J. L., and Pick, M. (2007). "Solar radio emissions," in Handbook of the Solar-Terrestrial Environment, eds Y. Kamide and A. Chian (Berlin; Heidelberg: Springer), 133-151.

Cane, H. V., and Erickson, W. C. (2005). Solar type II radio bursts and IP type II events. Astrophys. J. 623, 1180-1194. doi: 10.1086/428820

Carley, E. P., Baldovin, C., Benthem, P., Bisi, M. M., Fallows, R. A., Gallagher, P. T., et al. (2020a). Radio observatories and instrumentation used in space weather science and operations. J. Space Weather Space Clim. 10:7. doi: $10.1051 /$ swsc/2020007

Carley, E. P., Vilmer, N., Simões, P. J. A., and Ó Fearraigh, B. (2017). Estimation of a coronal mass ejection magnetic field strength using radio observations of gyrosynchrotron radiation. Astron. Astrophys. 608:A137. doi: 10.1051/0004-6361/201731368

Carley, E. P., Vilmer, N., and Vourlidas, A. (2020b). Radio observations of coronal mass ejection initiation and development in the low solar corona.

Casini, R., White, S. M., and Judge, P. G. (2017). Magnetic diagnostics of the solar corona: synthesizing optical and radio techniques. Space Sci. Rev. 210, 145-181. doi: 10.1007/s11214-017-0400-6

Chen, P. F. (2011). Coronal mass ejections: models and their observational basis. Living Rev. Solar Phys. 8:1. doi: 10.12942/lrsp-2011-1

Cliver, E. W., Dennis, B. R., Kiplinger, A. L., Kane, S. R., Neidig, D. F., Sheeley, N. R., et al. (1986). Solar gradual hard X-ray bursts and associated phenomena. Astrophys. J. 305, 920-935. doi: 10.1086/164306

Dagkesamanskii, R. D. (2009). The Pushchino Radio Astronomy Observatory of the P N Lebedev Physical Institute Astro Space Center: yesterday, today, and tomorrow. Phys. Uspekhi 52, 1159-1167. doi: 10.3367/UFNe.0179. 200911i.1225 
Fainberg, J., Evans, L. G., and Stone, R. G. (1972). Radio tracking of solar energetic particles through interplanetary space. Science 178, 743-745. doi: $10.1126 /$ science.178.4062.743

Fleishman, G., et al. (2020). Coherent emission. this volume.

Gary, D. E. (2020). Solar radio bursts. this volume.

Georgoulis, M. K., Nindos, A., and Zhang, H. (2019). The source and engine of coronal mass ejections. Philos. Trans. R. Soc. A Math. Phys. Eng. Sci. 377:20180094. doi: 10.1098/rsta.2018.0094

Gopalswamy, N., Akiyama, S., Yashiro, S., and Xie, H. (2017). A new technique to provide realistic input to CME forecasting models. Proc. Int. Astron. Union 13, 258-262. doi: $10.1017 / \mathrm{S} 1743921317011048$

Gopalswamy, N., Makela, P., and Yashiro, S. (2019). A catalog of type II radio bursts observed by wind/WAVES and their statistical properties. arXiv [Preprint]. arXiv:1912.07370. doi: 10.31401/SunGeo.2019.02.03

Gopalswamy, N., Xie, H., Makela, P., Akiyama, S., Yashiro, S., Kaiser, M. L., et al. (2010). Interplanetary shocks lacking type II radio bursts. Astrophys. J. 710, 1111-1126. doi: 10.1088/0004-637X/710/2/1111

Gopalswamy, N., Yashiro, S., Kaiser, M. L., Howard, R. A., and Bougeret, J.L. (2001). Radio signatures of coronal mass ejection interaction: coronal mass ejection cannibalism? Astrophys. J. Lett. 548, L91-L94. doi: 10.1086/ 318939

Gopalswamy, N., Yashiro, S., Xie, H., Akiyama, S., Aguilar-Rodriguez, E., Kaiser, M. L., et al. (2008). Radio-quiet fast and wide coronal mass ejections. Astrophys. J. 674, 560-569. doi: 10.1086/524765

Harrison, R. A., Davies, J. A., Barnes, D., Byrne, J. P., Perry, C. H., Bothmer, V., et al. (2018). CMEs in the heliosphere: I. A statistical analysis of the observational properties of CMEs detected in the heliosphere from 2007 to 2017 by STEREO/HI-1. Solar Phys. 293:77. doi: 10.1007/s11207-018$1297-2$

Jackson, B. V., et al. (2020). Interplanetary scintillation measurements. this volume. Jackson, B. V., Odstrcil, D., Yu, H. S., Hick, P. P., Buffington, A., Mejia-Ambriz, J. C., et al. (2015). The UCSD kinematic IPS solar wind boundary and its use in the ENLIL 3-D MHD prediction model. Space Weather 13, 104-115. doi: 10.1002/2014SW001130

Jensen, E. A., Hick, P. P., Bisi, M. M., Jackson, B. V., Clover, J., and Mulligan, T. (2010). Faraday rotation response to coronal mass ejection structure. Solar Phys. 265, 31-48. doi: 10.1007/s11207-010-9543-2

Kathiravan, C., Ramesh, R., and Nataraj, H. S. (2007). The post-coronal mass ejection solar atmosphere and radio noise storm activity. Astrophys. J. 656:L37. doi: $10.1086 / 512013$

Kerdraon, A., and Delouis, J.-M. (1997). “The nançay radioheliograph," in Coronal Physics from Radio and Space Observations, Vol. 483, ed G. Trottet (Berlin; Heidelberg: Springer), 192. doi: 10.1007/BFb0106458

Kilpua, E., Lugaz, N., Mays, M. L., and Temmer, M. (2019). Forecasting the structure and orientation of earthbound coronal mass ejections. Space Weather. 17, 1-29. doi: 10.1029/2018SW001944

Kilpua, E. K. J., Balogh, A., Steiger, R. V., and Liu, Y. D. (2017). Geoeffective properties of solar transients and stream interaction regions. Space Sci. Rev. 212, 1271-1314. doi: 10.1007/s11214-017-0411-3

Klein, K.-L., et al. (2020). Radio observations of solar energetic particles.

Kooi, J. E., Fischer, P. D., Buffo, J. J., and Spangler, S. R. (2017). VLA measurements of faraday rotation through coronal mass ejections. Solar Phys. 292:56. doi: 10.1007/s11207-017-1074-7

Krupar, V., Eastwood, J. P., Kruparova, O., Santolik, O., Soucek, J., Magdalenić, J., et al. (2016). An analysis of interplanetary solar radio emissions associated with a coronal mass ejection. Astrophys. J. Lett. 823(1):L5. doi: 10.3847/2041-8205/823/1/L5

Krupar, V., Santolik, O., Cecconi, B., Maksimovic, M., Bonnin, X., Panchenko, M., et al. (2012). Goniopolarimetric inversion using SVD: An application to type III radio bursts observed by STEREO. J. Geophys. Res. 117:A06101. doi: 10.1029/2011JA017333

Lazio, J., and Kasper, J. (2018). "The sun radio interferometer space experiment (SunRISE) mission concept," in 42nd COSPAR Scientific Assembly (Big Sky, MT).

Le Chat, G., Kasper, J. C., Cohen, O., and Spangler, S. R. (2014). Diagnostics of the solar corona from comparison between faraday rotation measurements and magnetohydrodynamic simulations. Astrophys. J. 789:163. doi: $10.1088 / 0004-637 \mathrm{X} / 789 / 2 / 163$
Leblanc, Y., Dulk, G. A., Vourlidas, A., and Bougeret, J.-L. (2001). Tracing shock waves from the corona to $1 \mathrm{AU}$ : Type II radio emission and relationship with CMEs. J. Geophys. Res. 106, 25301-25312. doi: 10.1029/2000JA000260

Lee, J., McClymont, A. N., Mikic, Z., White, S. M., and Kundu, M. R. (1998). Coronal currents, magnetic fields, and heating in a solar active region. Astrophys. J. 501:853. doi: 10.1086/305851

Lee, J., White, S. M., Gopalswamy, N., and Kundu, M. R. (1997). Signatures of coronal currents in microwave images. Solar Phys. 174, 175-190. doi: 10.1023/A:1004956422669

Leka, K. D., Park, S.-H., Kusano, K., Andries, J., Barnes, G., Bingham, S., et al. (2019). A comparison of flare forecasting methods. II. Benchmarks, metrics, and performance results for operational solar flare forecasting systems. Astrophys. J. 243:36. doi: 10.3847/1538-4365/ab2e12

Leske, R. A., Christian, E. R., Cohen, C. M. S., Cummings, A. C., Davis, A. J., Desai, M. I., et al. (2020). Observations of the 2019 April 4 solar energetic particle event at the parker solar probe. Astrophys. J. 246:35. doi: $10.3847 / 1538-4365 /$ ab5712

Li, W., Pober, J. C., Hazelton, B. J., Barry, N., Morales, M. F., Sullivan, I., et al. (2018). Comparing redundant and sky-model-based interferometric calibration: a first look with phase II of the MWA. Astrophys. J. 863:170. doi: 10.3847/1538-4357/aad3c3

Magdalenić, J., Marqué, C., Krupar, V., Mierla, M., Zhukov, A. N., Rodriguez, L., et al. (2014). Tracking the CME-driven Shock Wave on 2012 March 5 and radio triangulation of associated radio emission. Astrophys. J. 791:115. doi: 10.1088/0004-637X/791/2/115

Maia, D. J. F., Gama, R., Mercier, C., Pick, M., Kerdraon, A., and Karlický, M. (2007). The radio-coronal mass ejection event on 2001 April 15. Astrophys. J. 660, 874-881. doi: 10.1086/508011

Mäkelä, P., Gopalswamy, N., and Akiyama, S. (2018). Direction-finding analysis of the 2012 July 6 type II solar radio burst at low frequencies. Astrophys. J. 867:40. doi: 10.3847/1538-4357/aae2b6

Maksimovic, M., Bale, S. D., Vaivads, A., Krasnoselskikh, V., Chust, T., Balikhin, M., et al. (2007). "A radio and plasma wave experiment for the solar orbiter mission," in Second Solar Orbiter Workshop (Noordwijk: ESA Special Publication), 38.

Mason, J. P., Woods, T. N., Webb, D. F., Thompson, B. J., Colaninno, R. C., and Vourlidas, A. (2016). Relationship of EUV irradiance coronal dimming slope and depth to coronal mass ejection speed and mass. Astrophys. J. 830:20. doi: 10.3847/0004-637X/830/1/20

Matamoros, C. S., Klein, K. L., and Trottet, G. (2017). Microwave radio emissions as a proxy for coronal mass ejection speed in arrival predictions of interplanetary coronal mass ejections at 1 AU. J. Space Weather Space Clim. 7:A2. doi: $10.1051 / \mathrm{swsc} / 2016038$

McCauley, P. I., Cairns, I. H., White, S. M., Mondal, S., Lenc, E., Morgan, J., et al. (2019). The low-frequency solar corona in circular polarization. Solar Phys. 294:106. doi: 10.1007/s11207-019-1502-y

Mei, Y., Wang, F., Wang, W., Chen, L., Liu, Y., Deng, H., et al. (2018). GPUbased high-performance imaging for Mingantu spectral radioheliograph. Publ. Astron. Soc. Pac. 130:014503. doi: 10.1088/1538-3873/aa9608

Mejia-Ambriz, J. C., Villanueva-Hernandez, P., Gonzalez-Esparza, J. A., AguilarRodriguez, E., and Jeyakumar, S. (2010). Observations of interplanetary scintillation (IPS) using the Mexican array radio telescope (MEXART). Solar Phys. 265, 309-320. doi: 10.1007/s11207-010-9562-z

Mondal, S., Oberoi, D., and Vourlidas, A. (2019). Estimation of the physical parameters of a CME at high coronal heights using low frequency radio observations. arXiv [Preprint]. arXiv:1909.12041. doi: $10.3847 / 1538-4357 / \mathrm{ab} 7 \mathrm{fab}$

Morosan, D. E., Carley, E. P., Hayes, L. A., Murray, S. A., Zucca, P., Fallows, R. A., et al. (2019). Multiple regions of shock-accelerated particles during a solar coronal mass ejection. Nat. Astron. 3, 452-461. doi: 10.1038/s41550-019-0689-z

Mugundhan, V., Ramesh, R., Kathiravan, C., Gireesh, G. V. S., and Hegde, A. (2018). Spectropolarimetric observations of solar noise storms at low frequencies. Solar Phys. 293:41. doi: 10.1007/s11207-018-1260-2

Nindos, A., et al. (2020). Incoherent Emission.

Nindos, A., Kontar, E. P., and Oberoi, D. (2019). Solar physics with the Square Kilometre Array. Adv. Space Res. 63, 1404-1424. doi: 10.1016/j.asr.2018.10.023

Oberoi, D., and Lonsdale, C. J. (2012). Media responsible for Faraday rotation: a review. Radio Sci. 47:RS0K08. doi: 10.1029/2012RS004992 
Opgenoorth, H. J., Wimmer-Schweingruber, R. F., Belehaki, A., Berghmans, D., Hapgood, M., Hesse, M., et al. (2019). Assessment and recommendations for a consolidated European approach to space weather-as part of a global space weather effort. J. Space Weather Space Clim. 9:A37. doi: 10.1051/swsc/2019033

Patsourakos, S., Vourlidas, A., and Kliem, B. (2010). Toward understanding the early stages of an impulsively accelerated coronal mass ejection. SECCHI observations. Astron. Astrophys. 522:100. doi: 10.1051/0004-6361/200913599

Pick, M., Forbes, T. G., Mann, G., Cane, H. V., Chen, J., Ciaravella, A., et al. (2006). Multi-wavelength observations of CMEs and associated phenomena. Report of working group F. Space Sci. Rev. 123, 341-382. doi: 10.1007/s11214-006-9021-1

Pick, M., Malherbe, J.-M., Kerdraon, A., and Maia, D. J. F. (2005). On the Disk H $\alpha$ and radio observations of the 2003 October 28 flare and coronal mass ejection event. Astrophys. J. Lett. 631, L97-L100. doi: 10.1086/497137

Pohjolainen, S., Vilmer, N., Khan, J. I., and Hillaris, A. E. (2005). Early signatures of large-scale field line opening. Multi-wavelength analysis of features connected with a "halo" CME event. Astron. Astrophys. 434, 329-341. doi: 10.1051/0004-6361:20041378

Ramesh, R., Kathiravan, C., Barve, I. V., and Rajalingam, M. (2012a). High angular resolution radio observations of a coronal mass ejection source region at low frequencies during a solar eclipse. Astrophys. J. 744:165. doi: 10.1088/0004-637X/744/2/165

Ramesh, R., Lakshmi, M. A., Kathiravan, C., Gopalswamy, N., and Umapathy, S. (2012b). The location of solar metric type II radio bursts with respect to the associated coronal mass ejections. Astrophys. J. 752:107. doi: 10.1088/0004-637X/752/2/107

Robbrecht, E., Patsourakos, S., and Vourlidas, A. (2009). No trace left behind: STEREO observation of a coronal mass ejection without low coronal signatures. Astrophys. J. 701, 283-291. doi: 10.1088/0004-637X/701/1/283

Sasikumar Raja, K., Ramesh, R., Hariharan, K., Kathiravan, C., and Wang, T. J. (2014). An estimate of the magnetic field strength associated with a solar coronal mass ejection from low frequency radio observations. Astrophys. J. 796:56. doi: 10.1088/0004-637X/796/1/56

Savani, N. P., Vourlidas, A., Richardson, I. G., Szabo, A., Thompson, B. J., Pulkkinen, A., et al. (2017). Predicting the magnetic vectors within coronal mass ejections arriving at Earth: 2. Geomagnetic response. Space Weather 15, 441-461. doi: 10.1002/2016SW001458

Schrijver, C. J., Kauristie, K., Aylward, A. D., Denardini, C. M., Gibson, S. E., Glover, A., et al. (2015). Understanding space weather to shield society: a global road map for 2015-2025 commissioned by COSPAR and ILWS. Adv. Space Res. 55, 2745-2807. doi: 10.1016/j.asr.2015.03.023

Sukumar, S., Velusamy, T., Pramesh Rao, A., Swarup, G., and Bagri, D. S. (1988). Ooty synthesis radio telescope - design and performance. Bull. Astron. Soc. India 16, 93-110.

Sundkvist, D. J., Saint-Hilaire, P., Bain, H. M., Bale, S. D., Bonnell, J. W., Hurford, G. J., et al. (2016). "CURIE: cubesat radio interferometry experiment," in $A G U$ Fall Meeting Abstracts (San Fransisco, CA).

Tun, S. D., and Vourlidas, A. (2013). Derivation of the magnetic field in a coronal mass ejection core via multi-frequency radio imaging. Astrophys. J. 766:130. doi: $10.1088 / 0004-637 \mathrm{X} / 766 / 2 / 130$ van Haarlem, M. P., Wise, M. W., Gunst, A. W., Heald, G., McKean, J. P., Hessels, J. W. T., et al. (2013). LOFAR: The LOw-Frequency ARray. Astron. Astrophys. 556:A2. doi: 10.1051/0004-6361/201220873

Vourlidas, A. (2004). "Radio observations of coronal mass ejection4," in Solar and Space Weather Radiophysics: Current Status and Future Developments, Vol. 314, eds D. E. Gary and C. U. Keller (Dordrecht: Kluwer Academic Publishers), 223-242. doi: 10.1007/1-4020-2814-8_11

Vourlidas, A., and Bastian, T. S. (1996). Multiband VLA observations of solar active regions: implications for the distribution of coronal plasma. Astrophys. J. 466:1039. doi: 10.1086/177574

Vourlidas, A., Bastian, T. S., and Aschwanden, M. J. (1997). The structure of the solar corona above sunspots as inferred from radio, $\mathrm{x}$-ray, and magnetic field observations. Astrophys. J. 489:403. doi: 10.1086/304769

Vourlidas, A., Gary, D. E., and Shibasaki, K. (2006). Sunspot gyroresonance emission at $17 \mathrm{GHz}$ : a statistical study. Publ. Astron. Soc. Japan 58, 11-20. doi: $10.1093 /$ pasj/58.1.11

Vourlidas, A., Patsourakos, S., and Savani, N. P. (2019). Predicting the geoeffective properties of coronal mass ejections: current status, open issues and path forward. Philos. Trans. R. Soc. A Math. Phys. Eng. Sci. 377:20180096. doi: 10.1098/rsta.2018.0096

Webb, D. F., and Howard, T. A. (2012). Coronal mass ejections: observations. Living Rev. Solar Phys. 9:3. doi: 10.12942/lrsp-2012-3

White, S. M. (2005). "Coronal magnetic field measurements through gyroresonance emission," in Solar and Space Weather Radiophysics, Number 314 in Astrophysics and Space Science Library, eds D. E. Gary and C. U. Keller (Dordrecht: Springer), 89-113. doi: 10.1007/1-4020-2814-8_5

Willson, R. F. (2005a). Collaborative VLA, SOHO and RHESSI observations of evolving sources of energy release in the corona above active regions. Adv. Space Res. 35, 1813-1821. doi: 10.1016/j.asr.2005.04.081

Willson, R. F. (2005b). Very large array and SOHO observations of type I noise storms, large-scale loops and magnetic restructuring in the corona. Solar Phys. 227, 311-326. doi: 10.1007/s11207-0051104-8

Zucca, P., Morosan, D. E., Rouillard, A. P., Fallows, R., Gallagher, P. T., Magdalenic, J., et al. (2018). Shock location and CME 3D reconstruction of a solar type II radio burst with LOFAR. Astron. Astrophys. 615:A89. doi: 10.1051/0004-6361/201732308

Conflict of Interest: The authors declare that the research was conducted in the absence of any commercial or financial relationships that could be construed as a potential conflict of interest.

Copyright (c) 2020 Vourlidas, Carley and Vilmer. This is an open-access article distributed under the terms of the Creative Commons Attribution License (CC BY). The use, distribution or reproduction in other forums is permitted, provided the original author(s) and the copyright owner(s) are credited and that the original publication in this journal is cited, in accordance with accepted academic practice. No use, distribution or reproduction is permitted which does not comply with these terms. 